\title{
Near-Infrared Light-Enhanced Protease-Conjugated Gold Nanorods As A Photothermal Antimicrobial Agent For Elimination Of Exotoxin And Biofilms
}

This article was published in the following Dove Press journal: International Journal of Nanomedicine

\author{
Wei $\mathrm{Li}^{1,2}$ \\ Xu Geng' \\ Dongni Liu' \\ Zhengqiang Li $\mathbb{D}^{\prime}$
}

'Key Laboratory for Molecular Enzymology and Engineering of Ministry of Education, School of Life Science, Jilin University, Changchun I300I2, Jilin, People's Republic of China; ${ }^{2}$ Scientific Research Center, China-Japan Union Hospital, Jilin University, Changchun I30033, Jilin, People's Republic of China
Purpose: Treatment strategies to eliminate bacterial infections have long emphasized bacterial killing as a goal. However, bacteria secrete toxins that sustain chronic disease and dead cells release DNA that can promote the spread of antibiotic resistance even when viable cells are eradicated. Meanwhile, biofilms regulated by quorum-sensing system, protect bacteria and promote the development of antibiotic resistance. Thus, all of these factors underscore the need for novel antimicrobial therapeutic treatments as alternatives to traditional antibiotics. Here, a smart material was developed that incorporated gold nanorods and an adsorbed protease (protease-conjugated gold nanorods, PGs). When illuminated with near-infrared (NIR) light, PGs functioned to physically damage bacteria, prevent biofilm and exotoxin production, eliminate pre-existing biofilm and exotoxin, and inhibit bacterial quorum-sensing systems.

Methods: PGs were incubated with suspensions of Gram-negative (Escherichia coli) and Gram-positive (Staphylococcus aureus) bacteria followed by exposure to 808-nm NIR laser irradiation. Bacterial viability was determined using a colony-forming unit assay followed by an exploration of cell-damage mechanisms using transmission electron microscopy, scanning electron microscopy, agarose gel electrophoresis, and SDS-PAGE. Quantification of biofilm mass was performed using crystal violet staining. A commercial enterotoxin ELISA kit was used to test inhibitory and degradative effects of PGs on secreted exotoxin.

Results: Use of the remote-controlled antibacterial system reduced surviving bacterial populations to $3.2 \%$ and $2.1 \%$ of untreated control numbers for E. coli and $S$. aureus, respectively, and inhibited biofilm formation and exotoxin secretion even in the absence of NIR radiation. However, enhanced degradation of existing biofilm and exotoxin was observed when PGs were used with NIR laser irradiation.

Conclusion: This promising new strategy achieved both the reduction of viable microorganisms and elimination of biofilm and exotoxin. Thus, this strategy addresses the longignored issue of persistence of bacterial residues that perpetuate chronic illness in patients even after viable bacteria have been eradicated.

Keywords: photothermal therapy, synergistic effect, drug resistance, exotoxin, quorum sensing

\section{Introduction}

Infectious diseases induced by bacteria, a high priority health issue, cause millions of illnesses and deaths worldwide each year. ${ }^{1-3}$ Currently, antibiotic-based therapy is still the most common method used to treat bacterial infections. ${ }^{4}$ However, antibiotic overuse and abuse have led to increased bacterial resistance and poorer therapeutic
Key Laboratory for Molecular

Enzymology and Engineering of Ministry of Education, School of Life Science, Jilin University, Changchun 130012, Jilin,

People's Republic of China

Email Izq@jlu.edu.cn 
outcomes, with resulting increases in mortality observed in recent years. Bacterial resistance, the toughest problem hindering successful treatment of bacterial infections, occurs via four main mechanisms: 1) bacterial secretion of enzymes that inactivate antibacterial drugs, 2) alteration of antibiotic target sites that prevent binding of antibacterial drugs, 3) changes in permeability of bacterial outer membranes that prevents drug entry into bacteria, and 4) biofilm formation that surrounds and protects bacteria from antibiotic action. ${ }^{5,6}$ At the bacteria-host interface, biofilms are sessile communities of microbial cells that are cemented together by a bacterially produced exopolymeric matrix consisting of polysaccharides, proteins, DNA, and lipids. ${ }^{7-9}$ Biofilms are always associated with persistent infections and bacterially induced chronic inflammation. Once biofilms formed, bacteria within the exopolymeric matrix can develop greatly enhanced resistance to traditional antibiotics, due to acquired resistance, limited drug diffusion, and antibiotic inactivation. ${ }^{10-12}$ While antibiotics generally kill superficial and free-living bacteria effectively, they often cannot completely eradicate biofilmassociated infections, resulting in quick relapse after drug withdrawal. To overcome these problems, sustainable efforts have been made to develop new antimicrobial agents, including nanomaterials, antimicrobial peptides, hydrogels, etc., to prevent and combat bacterial invasion. ${ }^{13-17}$ Nevertheless, poor bioavailability, instability, and cytotoxicity have limited the clinical applicability of such smart materials.

In order to cause disease, pathogens deploy a series of virulence factors that protect them from the host innate immune response, enabling them to cross mucosal barriers, disseminate, and replicate in distant organs. ${ }^{18,19}$ Some of the most important bacterial virulence factors are toxins, including endotoxin present within the outer membrane of Gram-negative bacteria, as well as exotoxins secreted by diverse species of bacteria. Gram-positive bacteria and some Gram-negative bacteria can produce exotoxins, which consist mainly of protein and are characterized by low heat resistance, strong antigenicity, strong toxicity, and the ability to cause disease when present in only trace amounts. Moreover, exotoxins secreted into the extracellular milieu during bacterial growth and reproduction are closely associated with bacterial pathogenicity. Examples of exotoxins include the Pseudomonas aeruginosa pyocyanin that causes oxidative stress and an inflammatory reaction involving airway epithelial cells, Staphylococcus aureus enterotoxins that cause enteritis, and Escherichia coli enterotoxins that cause hemorrhagic diarrhea. ${ }^{20-22}$ Although many current studies aim to destroy biofilms to enhance bacterial clearance, harmful residual exotoxin effects have not yet been extensively addressed and warrant further study.

Biofilms and exotoxins are both regulated by quorumsensing (QS) systems, which are intercellular communication networks used by microorganisms to monitor local population densities and to control group-beneficial behaviors. $^{23-25}$ QS relies on the production, secretion, and perception of small diffusible signal molecules, referred to as autoinducers, which accumulate at concentrations that are directly proportional to cell density. Once a concentration of signal molecules reaches a threshold, the binding of signal molecules to receptors induces a series of gene expression changes that occur in an orchestrated manner. Such gene expression changes subsequently regulate bacterial population behaviors, including exotoxin secretion, biofilm formation, movement, luminescence, antibiotic production, pigmentation, nodulation, and other processes. ${ }^{26-28}$ In Gram-negative bacteria, the signal molecule is $\mathrm{N}$-acylated L-homoserine lactone, ${ }^{29}$ while in Gram-positive bacteria it is an auto-induced peptide (AIP). ${ }^{30}$ It is worth noting here that many bacteria secrete the same signal molecule to regulate the behaviors of bacteria belonging to other species. As for exotoxins in general, if the residual AIP signal is not removed, even after bacterial death, new biofilms and exotoxins will be produced quickly upon reinfection, even by a low number of bacteria. In view of the fact that biofilms, exotoxins, and signal molecules (of Gram-positive bacteria) are mainly based on proteins or polypeptides, proteases may serve as therapeutic agents to remove these factors due to its natural catalytic ability. However, bacterial mechanisms that inactivate proteases already exist that reduce protease effectiveness. Thus, methods to avoid protease inactivation are needed.

Recently, nanoparticles (NPs)-based photothermal therapy, currently used as a non-toxic therapeutic strategy to treat invasive cancer, may also be advantageous for antibacterial applications. ${ }^{31,32}$ For the treatment of bacterial infections, this strategy would be designed to expose infected intracorporal tissues and organs to relatively high temperatures that would selectively destroy pathogenic bacteria, while leaving host tissues undamaged. More specifically, this treatment would involve the administration of a microorganism-localized photosensitive nanoparticles that would be activated via illumination of infected tissues with light of a specific wavelength. ${ }^{33}$ To achieve deep tissue light penetration, the wavelength of 
the irradiated beam would be within the near-infrared (NIR) spectral region, between 700 and $1300 \mathrm{~nm}$, which is known as the "optical window" of biological tissue. Laser irradiation within the NIR spectral region would penetrate epidermal tissue with sufficient intensity and excellent spatial precision, resulting in radiation absorption by accumulated photosensitive nanomaterials within bacteria to induce local high temperature (hyperthermia). ${ }^{34}$ As an important point here, NIR light would have no adverse side effects on the human body, in contrast to known harmful effects of ultraviolet irradiation.

In this investigation, nanomaterials with high optical absorption within the NIR spectrum that incorporate only carbon-based nanostructures and gold-based NPs were used to provide photothermal therapy. ${ }^{35,36}$ Among these nanomaterials, gold nanoparticles (especially gold nanorods or GNRs) offer promise for use in biomedical applications, due to their unique optical properties, excellent biocompatibility, surface plasmon resonance effect, longterm stability, and ease of functionalization and bioconjugation. $^{37}$ In fact, GNRs have been shown to absorb NIR photoenergy more effectively than spherical gold nanoparticles. Due to their outstanding photothermal properties, which include a tunable excitation spectrum and ability to rapidly convert absorbed light energy into hyperthermia, GNRs should be suitable for the destruction of biofilms, bacterial killing, and denaturation of residual exotoxins and AIP. Compared to traditional infection control methods, this type of photothermal therapy has been previously demonstrated to have excellent selectivity with minimal side effects, since only cells containing nanomaterials with photothermal effect are damaged under-localized hyperthermia induced by focused NIR laser illumination. ${ }^{38}$ In addition, proteases can be easily conjugated to GNRs via any externally exposed protease cysteine residue using conventional Au-S chemistry. ${ }^{39}$ Indeed, after immobilization onto GNRs surfaces, great enhancement of protease stability has been observed that has been attributed to two factors: protection from bacterial inactivation and increased enzyme activity due to internal heat effects generated by GNRs. Here, a synergistic antibacterial effect was observed when modified complexes consisting of protease conjugated to GNRs were compared to free protease and GNRs alone. Collectively, these effects increase rates of enzymatic degradation of both exotoxin and biofilm proteins.

For all of the reasons listed above, protease-conjugated GNRs (PGs), an antimicrobial smart material consisting of a multifunctional complex that integrates the properties of protease (bromelain) with a gold nanorod scaffold, holds great promise as an antibacterial treatment option. By employing hyperthermia generated by conversion of NIR laser irradiation by GNRs, PGs not only achieve thermal elimination of biofilms and exotoxins, but also boost the activity of a conjugated mesophilic protease to achieve rapid degradation and removal of biofilm, exotoxin, and AIP proteins. Furthermore, the system described here exhibited a broad spectrum of antibacterial activity against both Gram-negative (Escherichia coli, E. coli) bacteria and gram-positive (Staphylococcus aureus, S. aureus) bacteria and has potential as an alternative treatment to antibiotics for treatment of bacterial infections.

\section{Materials And Methods GNRs Synthesis}

For GNRs preparation, $7.5 \mathrm{~mL}$ of $0.20 \mathrm{M}$ CTAB solution was added to $1.0 \mathrm{~mL}$ of $0.5 \mathrm{mM} \mathrm{HAuCl}_{4}$. Next, $0.6 \mathrm{~mL}$ of ice-cold $0.01 \mathrm{M} \mathrm{NaBH}_{4}$ was added dropwise to the stirred solution, resulting in the formation of a brownish-yellow solution. After continuous stirring of this seed solution for 2 mins, it was stored at $25^{\circ} \mathrm{C}$. Growth solution was prepared by mixing $237.5 \mathrm{~mL}$ of $0.1 \mathrm{M} \mathrm{CTAB}, 1.5 \mathrm{~mL}$ of $0.01 \mathrm{mM} \mathrm{AgNO}$, and $10 \mathrm{~mL}$ of $0.01 \mathrm{M} \mathrm{HAuCl}_{4}$ in a $500-\mathrm{mL}$ flask. Approximately, $1.6 \mathrm{~mL}$ of ascorbic acid $(0.1 \mathrm{M})$ was then slowly added dropwise to the mixture until it became colorless, after which additional ascorbic acid was added (a quarter of the total number of droplets to that point). Finally, $2 \mathrm{~mL}$ of the seed solution was added to the growth solution at $27-30^{\circ} \mathrm{C}$. The color of the solution changed gradually within 10-20 mins. During the entire process, the temperature of the growth medium was kept constant at $27-30^{\circ} \mathrm{C}$.

\section{PGs Composites Synthesis}

Synthesized GNRs (10-mL aliquots) were centrifuged at 12,500 rpm for 20 mins to remove excess CTAB surfactant. Each precipitate was dispersed in $8 \mathrm{~mL}$ of Milli-Q water after discarding the supernatant. Next, $0.2 \mathrm{M} \mathrm{K}_{2} \mathrm{CO}_{3}$ solution was added with stirring to adjust the $\mathrm{pH}$ to 9 . Following this step, $2.5 \mathrm{~mL}$ of $2 \mathrm{mg} / \mathrm{mL}$ protease was mixed with the GNRs solution with vigorous stirring at room temperature. Protease conjugation was allowed to proceed for $12 \mathrm{hrs}$. To remove excess protease, this procedure was then followed by centrifugation at $8000 \mathrm{rpm}$ for 10 mins. 


\section{Bacterial Culture And Antibacterial Experiments}

E. coli (ATCC 25922) and S. aureus (ATCC 25923) were obtained from ATCC (Manassas, VA, USA). Individual colonies of $E$. coli and $S$. aureus on solid Luria Bertani (LB) agar plates were transferred to $20 \mathrm{~mL}$ of liquid $\mathrm{LB}$ culture medium and grown at $37^{\circ} \mathrm{C}$ for $12 \mathrm{hrs}$ with agitation at $180 \mathrm{rpm}$. Next, bacteria were diluted with broth to $10^{6} \mathrm{CFU} / \mathrm{mL}$. Next, the bacterial suspension was mixed with different concentrations of PGs and mixtures were exposed to NIR illumination followed by spreading of each solution onto surfaces of plates containing solid medium. Plates were incubated in air for $24 \mathrm{hrs}$ at $37^{\circ} \mathrm{C}$ before counting numbers of bacterial colonies. Control experiments were performed in parallel without NIR illumination.

\section{Cleavage Of Intracellular Nucleic Acids And Proteins}

To investigate bacterial DNA degradation resulting from PGs treatment, extractions of E. coli and $S$. aureus genomic DNA were conducted using a bacterial genomic DNA purification kit before and after NIR laser irradiation. Assessment of nucleic acid cleavage was performed after addition of $50 \mu \mathrm{g} / \mathrm{mL}$ PGs followed by NIR illumination for 15 mins, after which characterization of nucleic acid cleavage products was performed using agarose gel electrophoresis and ethidium bromide staining. To obtain whole-cell proteins, E. coli and S. aureus cells were disrupted by sonication before or after NIR laser irradiation and soluble supernatants were obtained by centrifugation (10,000 rpm for $15 \mathrm{mins}$ ). Protein cleavage assays were performed after addition of $50 \mu \mathrm{g} / \mathrm{mL}$ PGs followed by NIR illumination for 15 mins prior to protein separation via SDS-PAGE and staining of gels with Coomassie blue R-250.

\section{Biofilm Formation By S. aureus}

For biofilm development, $10 \mu \mathrm{L}$ of stationary growth phase $S$. aureus bacterial culture, obtained after about 12 hrs of growth at $37^{\circ} \mathrm{C}$ in Tryptone Soy Broth (TSB) medium, was added to $990 \mu \mathrm{L}$ of biofilm minimal medium TSB medium (3\%, containing $1 \%$ glucose). For the development of $S$. aureus biofilms, PGs, protease, or GNRs were added into TSB medium containing 1-mL volumes of diluted $S$. aureus (final concentration: $\mathrm{OD}_{600 \mathrm{~nm}}=0.01$ ) in 24-well microtiter plates followed by incubation of microtiter plates at $37^{\circ} \mathrm{C}$ for $48 \mathrm{hrs}$. During incubation, spent medium was removed and replaced with fresh biofilm minimal medium every $24 \mathrm{hrs}$. After $48 \mathrm{hrs}$, each well was washed with PBS buffer under aseptic conditions to eliminate medium and unbound bacteria. Biofilms that had formed were visible at the bottoms of wells. Plates containing the same concentration of $S$. aureus with added biofilm minimal medium without added agents (TSB medium alone) were incubated in parallel to serve as the negative control (no treatment) group.

\section{Destruction And Inhibition Of S. aureus Biofilm Formation By The PGs-Based Antibacterial System}

Biofilms of $S$. aureus that grew in biofilm minimal medium in the presence of PGs, protease, or GNRs were exposed to various durations and intensities of NIR illumination in biofilm minimal media (TBS medium). After exposure, wells were washed once with PBS $(1.0 \mathrm{~mL})$ before staining with crystal violet $(1.0 \mathrm{~mL} ; 0.2 \%$ crystal violet, $1.9 \%$ ethanol, and $0.08 \%$ ammonium oxalate in PBS). For staining, plates were incubated in the benchtop for $20 \mathrm{mins}$ before washing wells with PBS $(2 \times 1.0 \mathrm{~mL})$. The amount of remaining crystal violet stain in each biofilm was quantified after addition of $100 \%$ ethanol $(1.0 \mathrm{~mL})$ followed by mixing and measurement of the $\mathrm{OD}_{590 \mathrm{~nm}}$ of the homogenized suspension. For the negative control, biofilms after growth for $48 \mathrm{hrs}$ in biofilm minimal media (TSB medium) without added treatments were analyzed in parallel as the negative control (no treatment) group.

\section{Clearance And Inhibition Of S. aureus Enterotoxins Using A PGs-Based Antibacterial System}

To inhibit secretion of $S$. aureus enterotoxins, PGs, protease, or GNRs were added to $S$. aureus cultures suspended in casamino acids-yeast extract (CAYE) medium in 24-well microtiter plates. Microtiter plates were then incubated at $37^{\circ} \mathrm{C}$ for $12 \mathrm{hrs}$. S. aureus cultures in CAYE medium without other additives were cultured in parallel with treated groups as the negative control group. Quantitative analysis of enterotoxins was performed using a commercial enterotoxin ELISA kit and results were compared to results obtained for the untreated control group. 


\section{Results And Discussion}

Preparation And Characterization Of PGs

GNRs were prepared as previously reported via a seedmediated surfactant-directed approach. ${ }^{40}$ The structural morphology of GNRs was initially characterized using transmission electron microscopy (TEM). As shown in Figure 1A, the average length of GNRs was about $32 \mathrm{~nm}$ and the average width was $7.8 \mathrm{~nm}$, for an aspect ratio of approximately 4.1. For PGs construction, specific concentrations of a commercial protease, bromelain, were added to separate GNRs solutions and incubated with stirring for $12 \mathrm{hrs}$. Bromelain is a sulfhydryl protease with an optimal reaction temperature of $50^{\circ} \mathrm{C}$. During conjugation to GNRs, thiol sulfur atoms of protease cysteine residues attach to surfaces of GNRs through the formation of polar covalent $\mathrm{Au}-\mathrm{S}$ bonds in a self-assembling manner. Protease-GNR complexes were characterized by UV-Vis spectroscopy (Figure 1B) and Fourier transform infrared spectroscopy (FTIR; Figure S1). UV-Vis spectroscopic analysis indicated that the longitudinal plasmon peak of
GNRs exhibited a red shift from $681 \mathrm{~nm}$ to $687 \mathrm{~nm}$ and the transverse plasmon peak exhibited a blue shift from 522 $\mathrm{nm}$ to $516 \mathrm{~nm}$, while the appearance of a characteristic peak at approximately $280 \mathrm{~nm}$ indicated successful peptide conjugation. Furthermore, our FTIR spectroscopy analysis indicated a weakening of the peak at $2600 \mathrm{~cm}^{-1}$ corresponding to cysteine thiol groups, thus confirming that $\mathrm{Au}-\mathrm{S}$ bonds had formed during protease attachment to GNRs surfaces. These data together reveal the successful immobilization of protease molecules onto GNRs to form PGs.

After PGs construction, we examined the photothermal conversion efficiency of PGs using 808-nm NIR laser irradiation at different PGs concentrations and power settings (Figure 1C and D). By adjusting PGs concentration and/or laser power, the exact-desired temperature (Figure 1C and D) could be obtained within the range of $20-66^{\circ} \mathrm{C}$. Meanwhile, conjugation-mediated changes in enzymatic activity were also investigated as previously reported, whereby enzymatic activity of PGs could be regulated within the range of $30-60^{\circ} \mathrm{C}$ by the adjustment of laser power (Figure S2).
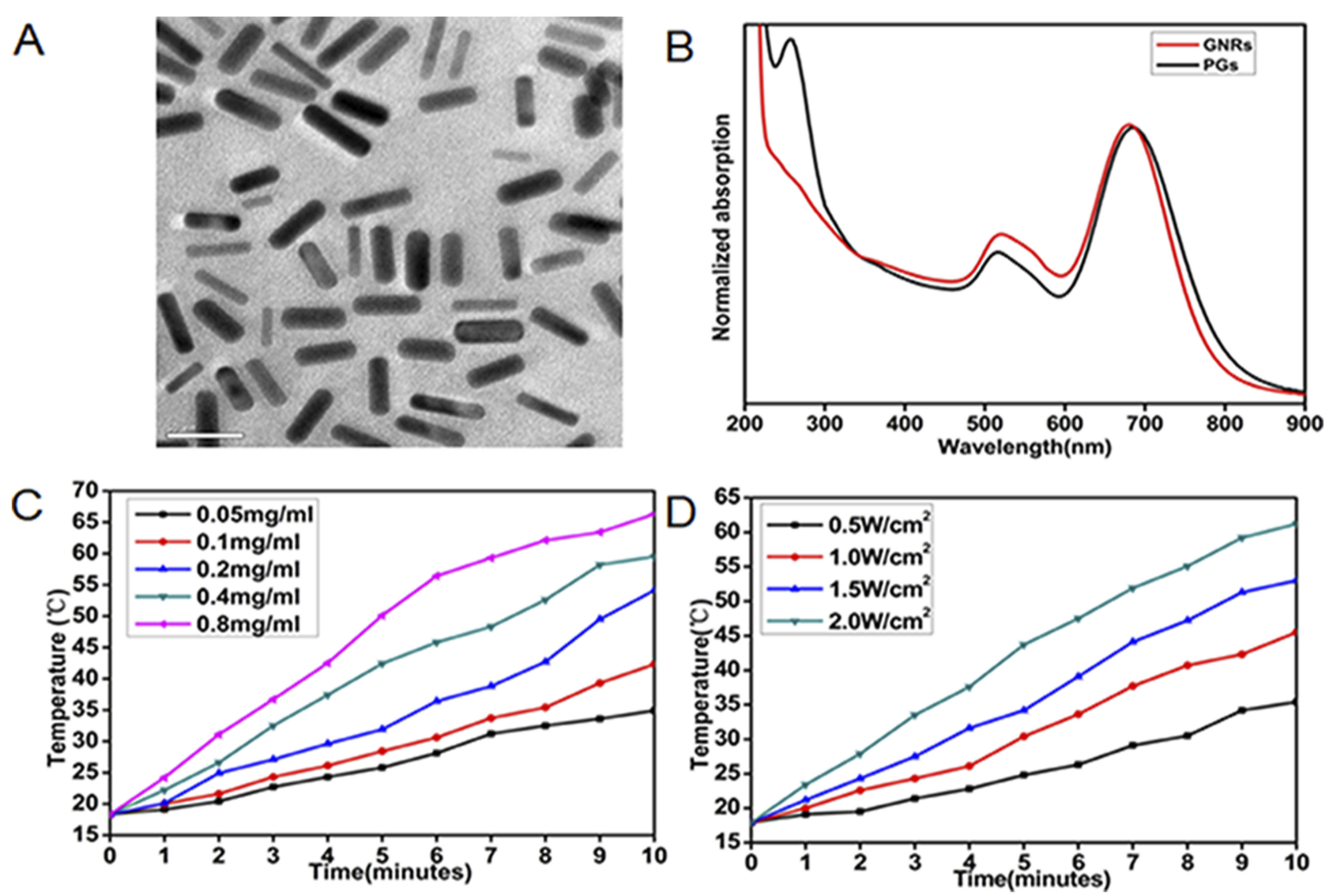

Figure I Characterization of GNRs and PGs. (A) TEM image of PGs. (B) UV-Vis absorption spectrum of GNRs and PGs. (C) NIR-induced heat generation of different concentrations of PGs aqueous solution at the same power density of $2 \mathrm{~W} \cdot \mathrm{cm}^{-2}$. (D) NIR-induced heat generation of $0.8 \mathrm{mg} \cdot \mathrm{mL}^{-1} \mathrm{PGs}$ at the different power densities. 
Intriguingly, compared to the enzymatic activity of free protease heated in a water bath to a given temperature, enhanced enzymatic activity was observed for PGs-conjugated protease upon NIR laser irradiation that maintained the same temperature. This result was consistent with previous reports indicating that internal heating mediated by the photothermal effect is a more efficient heat delivery method than that provided by diffusion-limited water bath-based heating. ${ }^{41,44}$ Moreover, the zeta potentials of the PGs complex and GNRs were $12.6 \pm 0.5 \mathrm{mV}$ and $-30.9 \pm 0.4 \mathrm{mV}$, respectively, demonstrating that PGs possess relatively higher colloidal stability and will not easily adsorb the majority of proteins in vivo but the negative-charged bacteria surface due to their greater degree of positive charge.

\section{Investigation Of Antibacterial Activity Of PGs}

To examine the antibacterial activity of PGs upon NIR laser irradiation, a common colony counting method was used here. Gram-negative bacteria (E. coli) and Gram-positive (S. aureus) were chosen as models to evaluate bactericidal efficiency. As control groups, different combinations of each individual antibacterial system component were prepared to identify if synergistic effects could be demonstrated. The various agents were mixed with bacterial preparations prior to NIR illumination (or no illumination for the control) followed by transfer of dilutions of the entire solutions to LB solid medium and subsequent incubation at $37^{\circ} \mathrm{C}$ for 12 hrs. As depicted in Figure 2A and B, bacterial cultures with added PGs with NIR laser irradiation exposure exhibited the strongest antimicrobial effects, with significant decreases in bacterial growth observed for both $E$. coli and $S$. aureus (96.8\% and 97.9\%, respectively). Minimal bactericidal concentrations of PGs for E. coli and $S$. aureus were $50 \mu \mathrm{g} / \mathrm{mL}$ and $40 \mu \mathrm{g} / \mathrm{mL}$, respectively, indicating that such a concentration could completely inactivate the bacteria and might lead to the loss of the pathogenicity. GNRs with NIR irradiation exhibited relatively weaker antibacterial effects against both microorganisms, with negligible antimicrobial effects even at a high GNRs concentration of $50 \mu \mathrm{g} / \mathrm{mL}$. Thus, PGsmediated hyperthermia contributed to observed bacterial cell death, while their GNRs component exhibited good biocompatibility. Intriguingly, the protease showed a certain degree of cytotoxicity against both $E$. coli and $S$. aureus, with bacterial densities of PGs-treated samples decreasing to approximately $56.8 \%$ and $67.4 \%$ that of untreated control, respectively. Thus, it was assumed that enhanced protease stability due to immobilization may protect the enzyme from inactivation. Consequently, such stabilization would be predicted to boost enzymatic degradation of bacterial surface transmembrane proteins or signal molecules (such as AIP) to further reduce bacterial viability, even at suboptimal temperatures. Moreover, according to the previous studies, photothermal effect regarded as an internal heating model could boost the activity of the conjugated enzymes, which may lead to a synergistic effect.

To discern the contribution of NIR irradiation to observed antibacterial effects, we further tested the growth kinetics of E. coli and $S$. aureus treated with PGs in the absence of NIR laser irradiation. PGs exhibited long-term inhibition efficiency compared with free bromelain due to enhanced protease stability, degradation of membrane protein, and inhibition of the QS system. As shown in Figure 2C and D, bromelain inhibited bacterial growth for $12 \mathrm{hrs}$ for both microorganisms. However, this inhibitory effect gradually disappeared by $24 \mathrm{hrs}$ due to bacterial inactivation of protease, a bacterial protective mechanism. In the experimental group, PGs inhibition of $E$. coli growth was only apparent until $24 \mathrm{hrs}$, after which the effect disappeared. Conversely, the inhibitory effect apparent in the growth curve of $S$. aureus was still clearly visible at $48 \mathrm{hrs}$. These data indicate that PGs are more cytotoxic to $S$. aureus than to E. coli and suggest that PGs may inhibit bacterial growth of $S$. aureus through disruption of QS.

With regard to QS effects, we should discuss the mechanisms underlying different antimicrobial effects of PGs on the two different strains of microorganisms studied here. S. aureus is a Gram-positive bacterium in which the structural components of cell wall contain a large amount of peptidoglycan. We assumed that protease would hydrolyze and degrade the short-peptide structure, resulting in the rupture of the cell wall and subsequent outflow of cell content and cell death. Therefore, any bactericidal components containing protease were predicted to cause more damage to $S$. aureus. Meanwhile, the QS signal molecule of $S$. aureus, a short peptide containing seven amino acids, may also be susceptible to protease degradation as a strategy to alter bacterial behavior and accelerate bacterial death. The other organism studied here, E. coli, is a Gram-negative bacterium with a cell wall which contains a larger amount of lipopolysaccharide than found in $S$. aureus. Lipopolysaccharide, also known as endotoxin, is toxic to the host and may be released after bacterial death or cell rupture. As endotoxin is heat-resistant, E. coli were predicted to be less sensitive to the photothermal effect 

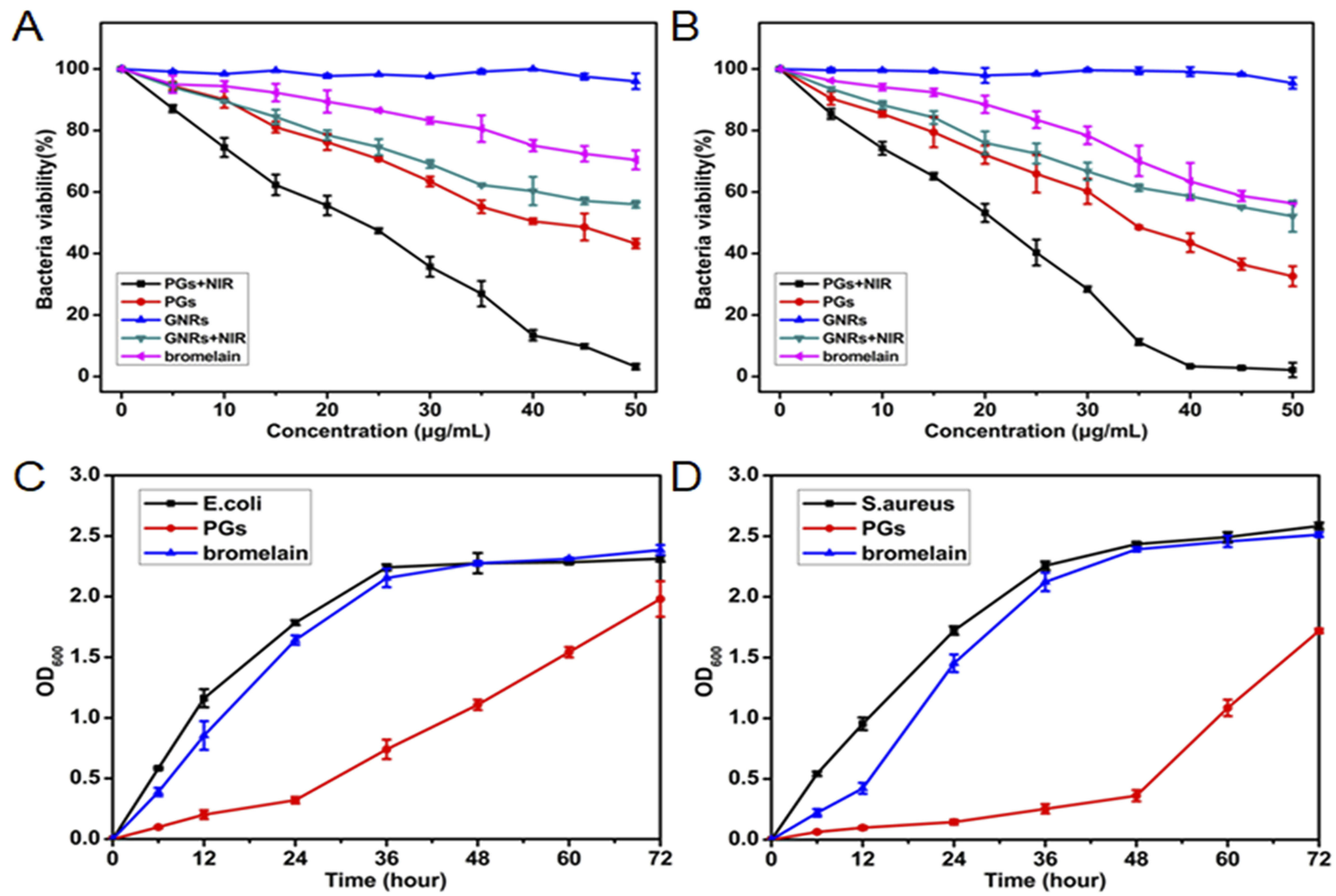

Figure 2 The bacterial viability of (A) E. coli and (B) S. aureus incubated with different concentrations of bromelain, GNRs or PGs upon NIR irradiation for 20 mins and an un-illuminated control. The growth curves of (C) E. coli and (D) S. aureus treated with $40 \mu \mathrm{g} \cdot \mathrm{mL}^{-1}$ of bromelain or PGs without illumination. Data points shown are the mean values \pm standard error of the mean (SEM) from three independent experiments.

when compared to $S$. aureus. When all of the aforementioned factors are considered together, PGs treatment should reduce the viability of $S$. aureus more dramatically than the effect predicted for E. coli.

To test these predictions, bacterial morphological changes induced by PGs complexes were studied using scanning electron microscopy (SEM) of E. coli and $S$. aureus with or without PGs treatment and NIR irradiation. As shown in Figure $3 \mathrm{~A}$ and $\mathrm{B}$, untreated E. coli was typically rod-shaped with clear edges and intact and smooth cell surfaces. However, after treatment with PGs with NIR laser irradiation, bacterial surfaces became rough and broken due to the destruction of bacterial membranes caused by hyperthermia and enzymatic degradation induced by the photothermal effect. As for cell walls of $S$. aureus, the results of SEM studies were similar to those for E. coli. S. aureus cells were sphere-shaped with intact surfaces before PGs treatment (Figure 3C). In the presence of both PGs and NIR laser irradiation, S. aureus cells became damaged and wrinkled (Figure 3D). These results indicate that the hyperthermia induction (via surface plasmon resonance) in conjunction with enzymatic degradation by conjugated protease served as an efficient and rapid bactericidal method that both damaged bacterial surfaces and degraded membrane proteins.

In addition, transmission electron microscope (TEM) experiments as a qualitative analysis method were conducted to explore the interaction between PGs and bacterial biofilms. PGs were observed to penetrate biofilms and adhere to bacterial surfaces, permitting thermal damage at an extremely close distance and further avoiding thermal damage to peripheral tissues (Figure S3). Moreover, the characteristics of S. aureus that is prone to form biofilms limited the diffusion of PGs via the holes in the biofilms, contributing to a potentially stronger thermal damage once upon NIR irradiation. In summary, all of these experiments demonstrate that PGs complexes possessed strong bactericidal properties against both Gram-negative and Gram-positive bacteria in this study. 

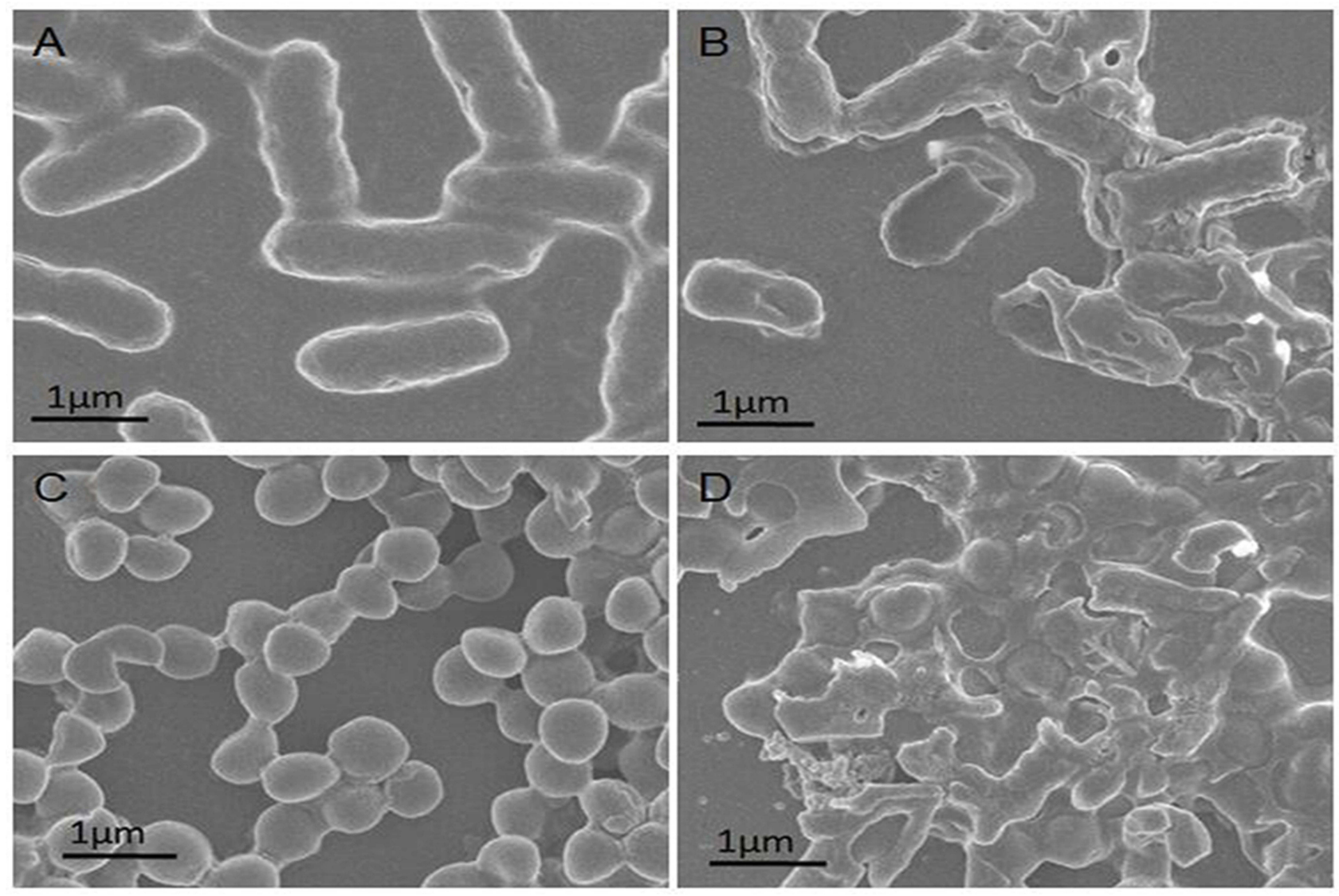

Figure 3 SEM images of (A) untreated E. coli, (B) E. coli treated with PGs $\left(50 \mu \mathrm{g} \cdot \mathrm{mL}^{-1}\right),\left(\right.$ C) untreated S. aureus and (D) S. aureus incubated with PGs (50 $\mu$ g $\left.\cdot \mathrm{mL}^{-1}\right)$ NIR illumination for 20 mins.

\section{Destruction Of Nucleic Acids And Intracellular Proteins}

Although bacteria can be killed by antibiotics or agents, remaining active bacterial proteins and genetic material, such as plasmids and bacterial DNA fragments, can still be absorbed and utilized by other living bacteria to induce genotypic and phenotypic changes in bystander bacteria. ${ }^{42}$ Gene transmission between bacteria is mainly achieved in three ways: transformation, conjugation, and transduction. ${ }^{42}$ Such gene dissemination leads to the spread of antibiotic resistance genes, exacerbating the problem of drug resistance. To assess whether PGs therapy could address this problem, bacterial samples without sonication were treated with PGs to detect degradation of whole-cell proteins as revealed by SDS-PAGE (Figure 4A) and degradation of genomic DNA as revealed using agarose gel electrophoresis (Figure 4B). The results showed that treatment with PGs at a concentration of $100 \mu \mathrm{g} / \mathrm{mL}$ with an NIR irradiation duration of 15 mins could effectively degrade bacterial proteins and nucleic acids in both $E$. coli and $S$. aureus un-sonicated cells.
From these results, we can conclude that PGs can serve as an efficient antibacterial smart material, since their generated hyperthermia together with enhanced protease activity effectively broke cell membranes to kill bacteria. In addition, PGsbased treatment also degraded intracellular proteins and genomic DNA, with the latter effect important for preventing horizontal gene transfer and spread of antibiotic resistance.

\section{Examination Of Biofilms Inhibition And Destruction}

Biofilms regulated by QS systems are difficult to eradicate, due to the fact that they protect the very bacteria that perpetuate their production. Moreover, biofilms contribute to antibiotic resistance and support chronic infections, such as those that afflict cystic fibrosis patients, while also interfering with wound healing and recovery from infection. Encouraged by the excellent photothermal and proteolytic effects of PGs for the destruction of proteins, we deployed this method to remove biofilms, which consist mainly of protein and depend on QS protein signals such as AIP for their formation. Specifically, 


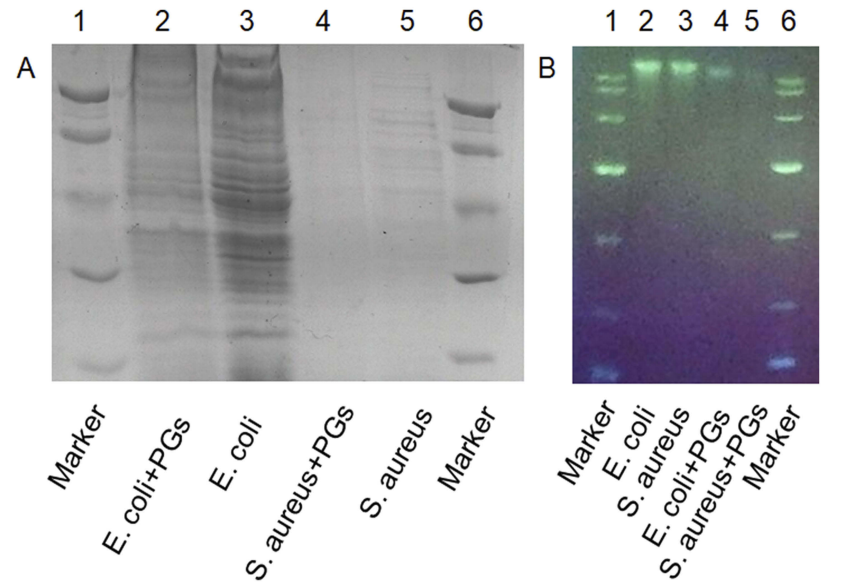

Figure 4 Cleavage of (A) whole proteins and (B) nucleic acids by PGs. E. coli and S. aureus were incubated with PGs under NIR light illumination for 20 mins prior to sonication.

both biofilms formation and destruction of bacteria were investigated using $S$. aureus as a model, since this bacterial species is a common clinical pathogen that forms biofilms on surfaces of biomaterials-containing implants. Using a standard crystal violet staining method to quantify the development of biofilm mass (Figure 5A), a weak inhibitory effect on biofilms formation was obtained using free protease treatment delivered in biofilm minimal medium. This weak effect likely resulted from bacterial inactivation of free protease. However, for protease conjugated to GNRs (PGs), formation of bacterial biofilms was almost completely inhibited by PGs, even without NIR illumination. Indeed, this inhibition was as observed in the face of continual secretion of new biofilm matrix protein and indicated that the conjugated protease exhibited enhanced stability as a benefit of conjugation. Meanwhile, AIP might be also degraded by PGs, further interrupting QS signals that would perpetuate biofilm formation. Thus, the excellent inhibitory effect of PGs on biofilms formation indicated that it exerted a sustainable and long-term effect that ultimately prevented pathogen growth.

With regard to pre-existing biofilms, experiments evaluating biofilm destruction by the PGs-based antibacterial system were also performed here. Indeed, the exposure of biofilms to GNRs or PGs each with NIR laser irradiation resulted in an obvious reduction in preformed biofilms (Figure 5B), with a removal of about $70.5 \%$ and $93.3 \%$ of biofilm mass, respectively. However, treatment with PGs without irradiation displayed only a moderate effect on biofilm destruction, with only a $16.8 \%$ reduction in biofilm mass, a value much smaller than the difference between the GNRs and PGs groups receiving NIR illumination. Thus, the PGs-mediated photothermal effect synergistically enhanced protease enzymatic activity. In contrast, biofilm in wells treated with GNRs (data not shown) or protease alone still showed clear intact biofilm bands, as did the negative control (no treatment) group. In summary, biofilms could be almost completely removed by $200 \mu \mathrm{g} / \mathrm{mL}$ PGs under $20 \mathrm{mins}$ NIR laser irradiation at an intensity of $2 \mathrm{~W} \cdot \mathrm{cm}^{-2}$.

From the abovementioned results, the photothermal effect mediated by PGs used with NIR laser irradiation has the potential to serve as an efficient remote-controlled
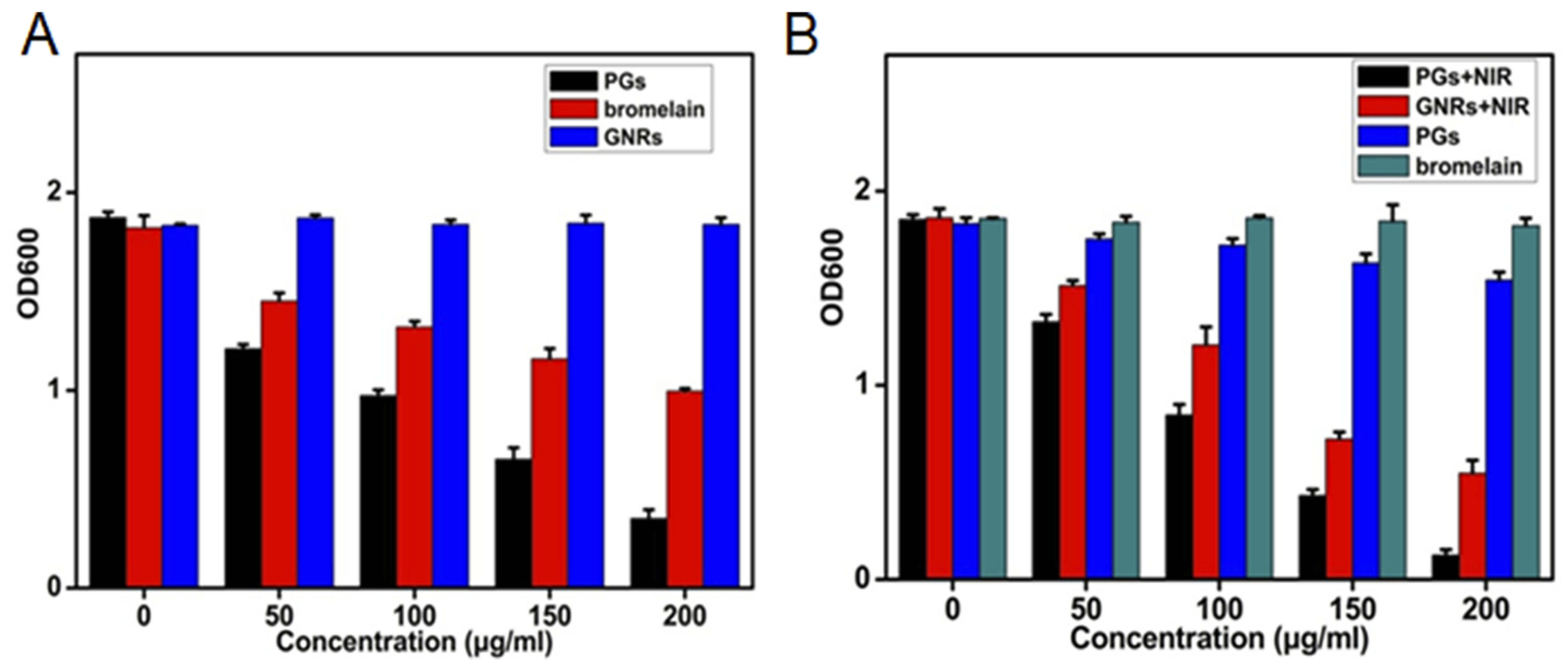

Figure 5 The efficacy of PGs on the (A) inhibition or (B) elimination of biofilms of S. aureus under NIR illumination for 30 mins or without NIR irradiation. Remaining biofilms were quantified by crystal violet staining. Data points shown are the mean values \pm SEM from three independent experiments. 
strategy for the removal of biofilms with minimal patient side-effects during clinical treatment. PGs exhibited both enhanced stability and protease activity through synergism stemming from protease immobilization that both protected the protease from degradation while enhancing enzymatic activity during hyperthermia induction. Moreover, this PGs-based antibacterial system could both prevent the formation of new biofilm and break down existing biofilm, with the combined use of PGs with NIR laser irradiation achieving bacterial killing within residual biofilms. Remarkably, the quantity of biofilms removed in the destruction experiment was much larger than that in the inhibition experiment, indicating that the photothermal effect was an efficient method for eliminating already existing biofilms. Furthermore, PGs achieved enhanced destruction of pre-formed and developing biofilms, as well as of signal AIP degradation and degradation of nucleic acids, proteins, and polysaccharides within the biofilm matrix. When taken together, these results demonstrate that the prevention of biofilm formation by PGs is a noteworthy and effective means of reducing the development of bacterial resistance.

\section{Examination Of Exotoxin Degradation}

Exotoxins can cause many diseases, such as sepsis, food poisoning, toxic shock syndrome, and diarrhea, etc. ${ }^{43}$ Although most recent studies have focused on the effective elimination of biofilms and on bacterial killing, they have neglected to address the persistence of highly toxic residual exotoxins that can lead to chronic illness. Due to its proteintargeting nature and heat-stability, a PGs-based antibacterial system could efficiently reduce pathogenicity caused by exotoxins. Herein, we used the exotoxin secreted by $S$. aureus (enterotoxin) as a model to examine the effect of PGs on inhibition and degradation of exotoxins. A commercial enterotoxin ELISA kit was employed to detect enterotoxin content with or without PGs treatment. As shown in Figure 6A, protease alone showed only a moderate inhibitory effect on enterotoxins secretion, due to degradation of the protease by bacterial extracellular enzymes. However, protease within PGs, even in the absence of NIR irradiation, could efficiently reduce enterotoxin levels during $72 \mathrm{hrs}$ of culture by degrading both newly secreted enterotoxins and the AIP QS signal.

We then tested the ability of PGs to remove existing enterotoxins. The results revealed that PGs effects were dose-dependent and achieved rapid enterotoxin clearance, with the removal of $86.5 \%$ of enterotoxin content after treatment with $200 \mu \mathrm{g} / \mathrm{mL}$ PGs after only 10 mins of NIR laser illumination (Figure 6B). Moreover, PGs with NIR irradiation destroyed thermolabile enterotoxins more effectively than did protease or PGs treatments alone. By contrast, in the absence of both the hyperthermal effect and conjugated protease enhancement, high enterotoxin content persisted. These results demonstrate that PGs can effectively inhibit or remove enterotoxin, which served here as a model for exotoxin. PGs thus have the potential to serve as treatments of chronic illnesses caused by
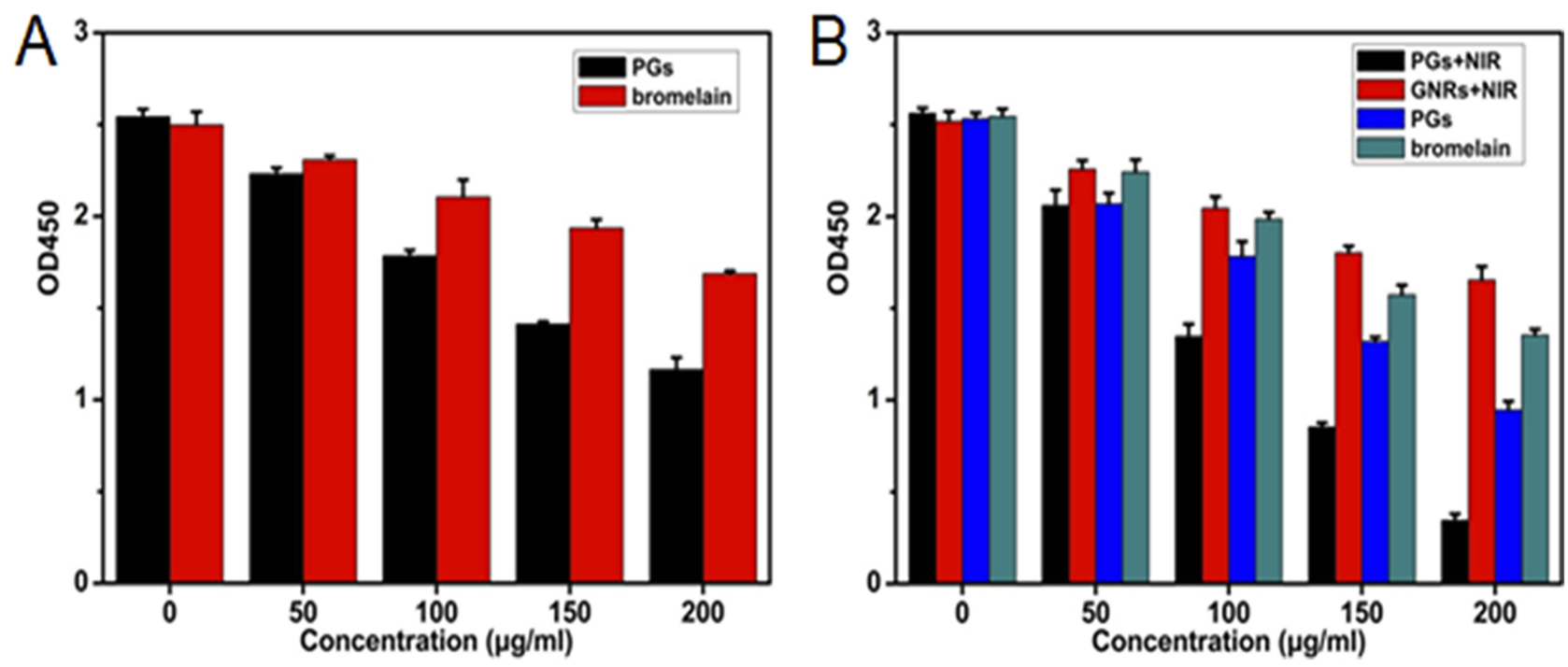

Figure 6 The efficacy of PGs on the (A) inhibition or (B) degradation of exotoxin of S. aureus upon NIR illumination for 30 mins or without NIR irradiation. The remaining enterotoxin was quantified by SE ELISA kit. Data points shown are the mean values \pm SEM from three independent experiments. 
exotoxin persistence in patients recovering from bacterial infections.

\section{Conclusion}

Due to the fact that biofilms and exotoxin production induced by bacterial QS systems can undermine treatment of infectious diseases, successful treatment outcomes require comprehensive therapy that incorporates multifunctional antibacterial agents. Based on this premise, we designed and synthesized PGs to serve as a novel smart antimicrobial agent. PGs exhibited not only broad antibacterial activity, but also achieved exotoxin clearance and biofilm removal when used with NIR illumination. The design of PGs integrated the unique NIR absorbance properties of GNRs with the efficient protein degradative ability of a protease. Internal hyperthermia generated by GNRs was used to enhance thermozyme activity to kill bacteria and prevent biofilm formation and destroy existing biofilm. When used in combination with NIR irradiation, conjugated protease within PGs was markedly more effective than free enzyme or GNRs alone for degrading enterotoxin and AIP. Notably, PGs could also degrade intracellular nucleic acids and proteins that contribute to the spread of antibiotic resistance. Taken together, our results indicate that PGs have potential uses as nanomedicines, with these offering new insights into future applications of proteases as antibacterial agents.

\section{Acknowledgment}

This work was supported by grants from Research Project of Science and Technology Department of Jilin Province (Grant No. 20180101265JC).

\section{Disclosure}

The authors report no conflicts of interest in this work.

\section{References}

1. Hook AL, Chang CY, Yang J, et al. Discovery of novel materials with broad resistance to bacterial attachment using combinatorial polymer microarrays. Adv Mater. 2013;25(18):2524-2527. doi:10.1002/ adma.201204936

2. Zhao Y, Chen Z, Chen Y, Xu J, Li J, Jiang X. Synergy of nonantibiotic drugs and pyrimidinethiol on gold nanoparticles against superbugs. J Am Chem Soc. 2013;135(35):12940-12943. doi:10.1021 /ja4058635

3. Pham VT, Truong VK, Quinn MD, et al. Graphene induces formation of pores that kill spherical and rod-shaped bacteria. ACS Nano. 2015;9 (8):8458-8467. doi:10.1021/acsnano.5b03368

4. Levy SB, Marshall B. Antibacterial resistance worldwide: causes, challenges and responses. Nat Med. 2004;10(12 Suppl):S122-S129. doi: $10.1038 / \mathrm{nm} 1145$
5. Dever LA, Dermody TS. Mechanisms of bacterial resistance to antibiotics. Arch Intern Med. 1991;151(5):886-895.

6. Blair JM, Webber MA, Baylay AJ, Ogbolu DO, Piddock LJ. Molecular mechanisms of antibiotic resistance. Nat Rev Microbiol. 2015;13 (1):42-51. doi:10.1038/nrmicro3380

7. Barraud N, Kardak BG, Yepuri NR, et al. Cephalosporin-3'-diazeniumdiolates: targeted NO-donor prodrugs for dispersing bacterial biofilms. Angew Chem Int Ed Engl. 2012;51(36):9057-9060. doi:10.1002/anie. 201202414

8. Durmus NG, Taylor EN, Kummer KM, Webster TJ. Enhanced efficacy of superparamagnetic iron oxide nanoparticles against antibiotic-resistant biofilms in the presence of metabolites. Adv Mater. 2013;25(40):5706-5713. doi:10.1002/adma.201302627

9. Li Y 1, Fukushima K, Coady DJ, et al. Broad-spectrum antimicrobial and biofilm-disrupting hydrogels: stereocomplex-driven supramolecular assemblies. Angew Chem Int Ed Engl. 2013;52(2):674-678. doi:10.1002/anie.201206053

10. Liu R, Chen X, Falk SP, Masters KS, Weisblum B, Gellman SH. Nylon3 polymers active against drug-resistant candida albicans biofilms. $J$ Am Chem Soc. 2015;137(6):2183-2186. doi:10.1021/ja512567y

11. Yang X, Yang J, Wang L, et al. Pharmaceutical intermediate-modified gold nanoparticles: against multidrug-resistant bacteria and woundhealing application via an electrospun scaffold. ACS Nano. 2017;11 (6):5737-5745. doi:10.1021/acsnano.7b01240

12. Zhao Y, Tian Y, Cui Y, Liu W, Ma W, Jiang X. Small moleculecapped gold nanoparticles as potent antibacterial agents that target gram-negative bacteria. J Am Chem Soc. 2010;132(35):12349-12356. doi:10.1021/ja1028843

13. Lee J, Mahendra S, Alvarez PJ. Nanomaterials in the construction industry: a review of their applications and environmental health and safety considerations. ACS Nano. 2010;4(7):3580-3590. doi:10.1021/ nn100866w

14. Akhavan O, Ghaderi E. Toxicity of graphene and graphene oxide nanowalls against bacteria. ACS Nano. 2010;4(10):5731-5736. doi:10.1021/ nn101390x

15. Tu Y, Lv M, Xiu P, et al. Destructive extraction of phospholipids from Escherichia coli membranes by graphene nanosheets. Nat Nanotechnol. 2013;8(8):594-601. doi:10.1038/nnano.2013.125

16. Zhao J, Deng B, Lv M, et al. Graphene oxide-based antibacterial cotton fabrics. Adv Healthc Mater. 2013;2(9):1259-1266. doi:10. 1002/adhm.201200437

17. Lucky SS, Soo KC, Zhang Y. Nanoparticles in photodynamic therapy. Chem Rev. 2015;115(4):1990-2042. doi:10.1021/cr5004198

18. Cavaillon JM. Exotoxins and endotoxins: inducers of inflammatory cytokines. Toxicon. 2018;149:45-53. doi:10.1016/j.toxicon.2017.10. 016

19. Ramachandran G. Gram-positive and gram-negative bacterial toxins in sepsis: a brief review. Virulence. 2014;5(1):213-218. doi:10.4161/ viru. 27024

20. Leshem Y, Pastan I. Pseudomonas exotoxin immunotoxins and antitumor immunity: from observations at the patient's bedside to evaluation in preclinical models. Toxins (Basel). 2019;11(1):E20. doi:10.3390/toxins 11010020

21. Spaulding AR, Salgado-Pabón W, Kohler PL, Horswill AR, Leung DY, Schlievert PM. Staphylococcal and streptococcal superantigen exotoxins. Clin Microbiol Rev. 2013;26(3):422-447. doi:10.1128/ CMR.00104-12

22. Gonzales-Siles L, Sjöling Å. The different ecological niches of enterotoxigenic Escherichia coli. Environ Microbiol. 2016;18(3):741-751. doi:10.1111/1462-2920.13106

23. Doğaner BA, Yan LKQ, Youk H. Autocrine signaling and quorum sensing: extreme ends of a common spectrum. Trends Cell Biol. 2016;26(4):262-271. doi:10.1016/j.tcb.2015.11.002

24. Papenfort K, Bassler BL. Quorum sensing signal-response systems in gram-negative bacteria. Nat Rev Microbiol. 2016;14(9):576-588. doi:10.1038/nrmicro.2016.89 
25. Carnes EC, Lopez DM, Donegan NP, et al. Confinement-induced quorum sensing of individual Staphylococcus aureus bacteria. Nat Chem Biol. 2010;6(1):41-45. doi:10.1038/nchembio.264

26. O'Loughlin CT, Miller LC, Siryaporn A, Drescher K, Semmelhack MF, Bassler BL. A quorum-sensing inhibitor blocks Pseudomonas aeruginosa virulence and biofilm formation. Proc Natl Acad Sci U S A. 2013;110(44):17981-17986. doi:10.1073/pnas.1316981110

27. Hong SH, Hegde M, Kim J, Wang X, Jayaraman A, Wood TK. Synthetic quorum-sensing circuit to control consortial biofilm formation and dispersal in a microfluidic device. Nat Commun. 2012;3:613. doi:10.1038/ncomms1616

28. Tsuchikama K, Gooyit M, Harris TL. Glycation reactivity of a quorum-sensing signaling molecule. Angew Chem Int Ed Engl. 2016;55(12):4002-4006. doi:10.1002/anie.201511911

29. Fuqua C, Greenberg EP. Listening in on bacteria: acyl-homoserine lactone signalling. Nat Rev Mol Cell Biol. 2002;3(9):685-695. doi:10.1038/nrm907

30. Tal-Gan Y, Ivancic M, Cornilescu G, Yang T, Blackwell HE. Highly stable, amide-bridged autoinducing peptide analogues that strongly inhibit the AgrC quorum sensing receptor in Staphylococcus aureus. Angew Chem Int Ed Engl. 2016;55(31):8913-8917. doi:10.1002/ anie. 201602974

31. Millenbaugh NJ, Baskin JB, DeSilva MN, Elliott WR, Glickman RD. Photothermal killing of Staphylococcus aureus using antibody-targeted gold nanoparticles. Int J Nanomed. 2015;10:1953-1960. doi:10.2147/IJN.S76150

32. Kim SH, Kang EB, Jeong CJ, Sharker SM, In I, Park SY. Light controllable surface coating for effective photothermal killing of bacteria. ACS Appl Mater Interfaces. 2015;7(28):15600-15606. doi:10.1021/acsami.5b04321

33. Karahan HE, Wiraja C, Xu C. Graphene materials in antimicrobial nanomedicine: current status and future perspectives. Adv Healthc Mater. 2018;7(13):e1701406. doi:10.1002/adhm.201701406

34. Hu D, Li H, Wang B. Surface-adaptive gold nanoparticles with effective adherence and enhanced photothermal ablation of methicillin-resistant Staphylococcus aureus biofilm. ACS Nano. 2017;11 (9):9330-9339. doi:10.1021/acsnano.7b04731
35. Ghosh S, Dutta S, Gomes E, et al. Increased heating efficiency and selective thermal ablation of malignant tissue with DNA-encased multiwalled carbon nanotubes. ACS Nano. 2009;3(9):2667-2673. doi:10.1021/nn900368b

36. Hirsch LR, Stafford RJ, Bankson JA, et al. Nanoshell-mediated nearinfrared thermal therapy of tumors under magnetic resonance guidance. Proc Natl Acad Sci U S A. 2003;100(23):13549-13554. doi:10.1073/ pnas. 2232479100

37. Krol S, Macrez R, Docagne F, et al. Therapeutic benefits from nanoparticles: the potential significance of nanoscience in diseases with compromise to the blood brain barrier. Chem Rev. 2013;113 (3):1877-1903. doi:10.1021/cr200472g

38. Liu D, Li W, Jiang X, et al. Using near-infrared enhanced thermozyme and $s c F v$ dual-conjugated Au nanorods for detection and targeted photothermal treatment of Alzheimer's disease. Theranostics. 2019;9(8):2268-2281. doi:10.7150/thno.30649

39. Hinterwirth H, Kappel S, Waitz T, Prohaska T, Lindner W, Lämmerhofer M. Quantifying thiol ligand density of self-assembled monolayers on gold nanoparticles by inductively coupled plasma-mass spectrometry. ACS Nano. 2013;7(2):1129-1136. doi:10.1021/nn306024a

40. Yang X, Liu X, Liu Z, Pu F, Ren J, Qu X. Near-infrared light-triggered, targeted drug delivery to cancer cells by aptamer gated nanovehicles. Adv Mater. 2012;24(21):2890-2895. doi:10.1002/adma.201104797

41. Blankschien MD, Pretzer LA, Huschka R, Halas NJ, Gonzalez R, Wong MS. Light-triggered biocatalysis using thermophilic enzymegold nanoparticle complexes. ACS Nano. 2013;7(1):654-663. doi:10.1021/nn3048445

42. Rasko DA, Sperandio V. Anti-virulence strategies to combat bacteriamediated disease. Nat Rev Drug Discov. 2010;9(2):117-128. doi: $10.1038 / \mathrm{nrd} 3013$

43. Dinges MM, Orwin PM, Schlievert PM. Exotoxins of Staphylococcus aureus. Clin Microbiol Rev. 2000;13(1):16-34. doi:10.1128/cmr.13. 1.16-34.2000

44. Li W, Liu D, Xu G, Li Z, Gao R. Real-time regulation of catalysis by remote-controlled enzyme-conjugated gold nanorod composites for aldol reaction-based applications. Catal Sci Technol. 2019;9:22212230. doi:10.1039/C9CY00167K
International Journal of Nanomedicine

\section{Publish your work in this journal}

The International Journal of Nanomedicine is an international, peerreviewed journal focusing on the application of nanotechnology in diagnostics, therapeutics, and drug delivery systems throughout the biomedical field. This journal is indexed on PubMed Central, MedLine, CAS, SciSearch ${ }^{\circledR}$, Current Contents ${ }^{\mathbb{R}} /$ Clinical Medicine, $^{-}$
Journal Citation Reports/Science Edition, EMBase, Scopus and the Elsevier Bibliographic databases. The manuscript management system is completely online and includes a very quick and fair peer-review system, which is all easy to use. Visit http://www.dovepress.com/ testimonials.php to read real quotes from published authors 